\title{
Symptomatic versus asymptomatic pyeloplasties: A single institution review
}

\author{
Peter D. Metcalfe, MD, BSc; Mark Assmus, BSc; Darcie Kiddoo, MD, FRCSC
}

Division of Pediatric Surgery, Stollery Children's Hospital, Edmonton, AB

Cite as: Can Urol Assoc J 2014;8(11-12):428-31. http://dx.doi.org/10.5489/cuai.2271 Published online December 15, 2014.

\section{Abstract}

Introduction: Historically, pyeloplasties have been performed after symptoms and radiographic confirmation of an ureteropelvic junction obstruction (UPJO). However, with prenatal ultrasonography, the approach to patients has fundamentally changed. Increasingly, patients are diagnosed and treated before the advent of morbidity, based on imaging findings alone. However, optimum screening strategies and thresholds for intervention vary significantly, are controversial, and are not founded on outcome-based evidence. We examined all pyeloplasties performed at our institution and reviewed their indication for surgery. We hypothesized that, despite ubiquitous screening for UPJO, most pyeloplasties had been performed secondary to symptoms and did not benefit from antenatal screening.

Methods: A retrospective chart review was performed of all pyeloplasties performed at the Stollery Children's Hospital, Edmonton, Alberta, over the past 8 years. Patients were categorized according to indication for surgery: symptomatic or asymptomatic.

Results: Most (60\%) of our pyeloplasties were performed for symptomatic indications. Furthermore, $12 \%$ of these patients had antenatally detected hydronephrosis that was thought to have resolved spontaneously during follow-up. Of our symptomatic patients, 37\% were undergoing surveillance with the expectation for spontaneous resolution. Of the 29 patients who underwent pyeloplasty, 8 suffered a preoperative loss of function on renal scans; however, only $50 \%$ returned to within $90 \%$ of their original function.

Conclusion: Despite active surveillance of antenatally detected hydronephrosis, most pyeloplasties at our institution were performed for de-novo symptoms. We believe that this simple observation reinforces that our current surveillance strategies are unable to predict and eliminate all morbidity from UPJO.

\section{Introduction}

Pyeloplasty is a fundamental piece of the pediatric urologists' armamentarium, with the definite goals of preserving renal function and preventing morbidity secondary to ureteropelvic junction obstruction (UPJO). Classically, patients present with Dietl's crisis, infections, or kidney stones; however, prenatal assessments aim to identify significant UPJO and prevent deleterious consequences.

The hypothesis for this study is that despite nearly ubiquitous screening and excellent follow-up, a significant number of our patients presented with previously normal imaging and underwent surgery for a symptomatic UPJO.

Therefore, we have reviewed all of our patients who have undergone a pyeloplasty. We have compared those that had surgery secondary to abnormal imaging, and had remained asymptomatic, to patients who underwent a pyeloplasty secondary to symptoms from their UPJO. We intentionally did not review all of our patients with antenatal hydronephrosis $(\mathrm{ANH})$, as this review was not intended to review overall rates of intervention, efficacy of our screening, or relative risks.

\section{Methods}

After University institutional review board approval, we performed a retrospective chart review of all pediatric pyeloplasties at the Stollery Children's Hospital, Edmonton, Alberta, from January 2007 to June 2013.

Data from all 72 procedures were classified according to the indication for surgery. Patients who had been followed secondary to $\mathrm{ANH}$ and who underwent surgery secondary to radiographic finding (i.e., decreased function, poor drainage, worsening hydronephrosis) were considered assymptomatic.

Symptomatic patients were those patients who presented with pain (including Dietl's crisis), urinary tract infection, pyelonephritis, stones, renal insufficiency, failure to thrive, abscess, or sepsis. In this group, we included patients who did not have any evidence of ANH, those who had "resolution" of their imaging while on surveillance, and those who developed symptoms while actively on surveillance.

Continuous variables were expressed as a mean and a range, while proportions were used for categorical variables. Statistical analysis was peformed using a one-tailed distribu- 
tion, heteroscedastic t-test. A $p$-value $<0.05$ was considered statistically significant.

\section{Results}

\section{Population characteristics}

In total, 68 patients underwent 72 pyeloplasties at the Stollery Children's Hospital from 2007 to 2013. Of these, 60\% (43/72) of pyeloplasties had symptomatic indications for surgery, while $40 \%$ (29/72) of renal units were asymptomatic and undergoing radiographic surveillance for their $\mathrm{ANH}$.

Overall, 60\% (41/68) of patients had a history of ANH and had been monitored by their pediatric urologist. Generally, patients with mild (Society for Fetal Urology [SFU] grade $1+2$ ) hydronephrosis were followed semi-annually and annually with ultrasound until resolution of their hydronephrosis. Higher grades were followed every 3 to 6 months with an ultrasound. MAG-3 scans were employed to determine initial function, to assess for deterioration of function, and to attempt to quantify drainage. Five patients had resolved their hydronephrosis and were discharged from active surveillance (Table 1). Resolution was defined as a single ultrasound with no hydronephrosis.

Of the total 68 patients, $96 \%(n=65)$ had pre-surgical MAG-3 scans, with a mean ipsilateral function of $41 \%$ $(10 \%-50 \%)$. Moreover, $32 \%$ (23/72) of cases had equivalent bilateral function (i.e., kidneys were considered equivalent if the ipsilateral function was $>40 \%$, to account for errors and variation of the MAG-3). Of these 65 patients with presurgical MAG-3 scans, $12 \%(n=8)$ of patients had an initial ipsilateral function less than $25 \%$.

All patients did well postoperatively, with $100 \%$ symptom resolution and $80 \%$ resolution of hydronephrosis on ultrasound imaging at 1-year post-pyeloplasty in both groups. There have not been any re-do pyeloplasties, and 1 patient has responded well to endoscopic dilation.

\section{Comparison of symptomatic vs. asymptomatic screening indicated patients}

Symptomatic patients underwent surgery at a mean age of 86 months, while asymptomatic patients underwent surgery at 27 months $(p<0.001)$ (Table 2$)$. There was no statistical

\begin{tabular}{lc}
\hline Table 1. Characteristics of all 72 pyeloplasties \\
\hline Procedure characteristics & No. of all 72 cases \\
\hline Symptomatic & $60 \%(43)$ \\
Laparoscopic & $19(14)$ \\
Open & $81(58)$ \\
Right kidney & $36(26)$ \\
Left kidney & $64(46)$ \\
\hline
\end{tabular}

difference between average time to ultrasound resolution and the average length of follow-up or average time between serial ultrasound between the 2 groups.

\section{Population with symptomatic UPJ0 indications for surgery}

Of the 43 symptomatic patients, $52 \%(22 / 43)$ had no history of $\mathrm{ANH}$, and $37 \%$ (16/43) developed symptoms, while undergoing surveillance of their $\mathrm{ANH}$. Of these 16 patients who were undergoing observation, 10 of them had SFU grade 4 hydronephrosis, and 3 patients had ipsilateral renal function $<40 \%$.

Interestingly, $12 \%(5 / 43)$ had been discharged from surveillance of their $\mathrm{ANH}$, due to resolution of their hydronephrosis on ultrasound. The 5 patients who experienced previous resolution of their $\mathrm{ANH}$ presented back to their urologist with symptoms a mean 31 months after their last clinic appointment (and discharged from surveillance) with symptomatic UPJO (Fig. 1, Table 3).

\section{Population with asymptomatic screening indications for surgery}

The surgical indications for the 29 patients in the asymptomatic group included: decrease in renal function on MAG-3 $(n=8)$; worsening hydronephrosis on ultrasound $(n=14)$; and lack of improvement after a period of observation $(n=7)$ The mean duration of follow-up in this group was 81 months, and mean SFU grade was 3.

Of the 27 asymptomatic patients who were being followed with serial MAG-3 scans, $30 \%$ (8/27) suffered $a \geq 10 \%$ decrease in function. However, only $50 \%(4 / 8)$ of these patients returned to within $90 \%$ of their initial differential function after pyeloplasty. The mean duration of postoperative follow-up was 13.8 months. For these 8 asymptomatic patients, their mean preoperative SFU grade was 2-4, with most having grade 3 or worse (2/10 grade $3,4 / 10$ grade

\begin{tabular}{lccc}
\hline \multicolumn{4}{l}{$\begin{array}{l}\text { Table 2. Comparison of patients who had a UPJO repair } \\
\text { secondary to symptoms vs. asymptomatic screening }\end{array}$} \\
\hline $\begin{array}{l}\text { Symptomatic } \\
\text { patients }\end{array}$ & $\begin{array}{c}\text { Asymptomatic } \\
\text { patients }\end{array}$ & p value \\
\hline $\begin{array}{l}\text { Age at surgery (months) } \\
\text { Age at first MAG-3 } \\
\text { (months) }\end{array}$ & 86 & 27 & $<0.001$ \\
$\begin{array}{l}\text { Time to ultrasound } \\
\text { resolution (months) }\end{array}$ & 77 & 16 & $<0.001$ \\
$\begin{array}{l}\text { Length of follow-up } \\
\begin{array}{l}\text { Time between serial } \\
\text { ultrasound }\end{array}\end{array}$ & 18 & 10 & 0.15 \\
$\begin{array}{l}\text { Average pre-surgical } \\
\text { MAG-3 function (\%) }\end{array}$ & 11 & 22 & 0.16 \\
$\begin{array}{l}\text { Average SFU hydro } \\
\text { grade pre-surgery }\end{array}$ & 42.7 & 10 & 0.34 \\
\hline UPJO: ureteropelvic junction obstruction; SFU: Society for Fetal Urology. & \\
\hline
\end{tabular}




\section{All pyeolplasties}

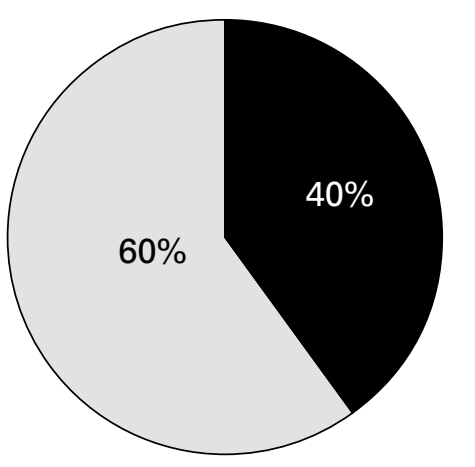

\section{Symptomatic pyeolplasty}

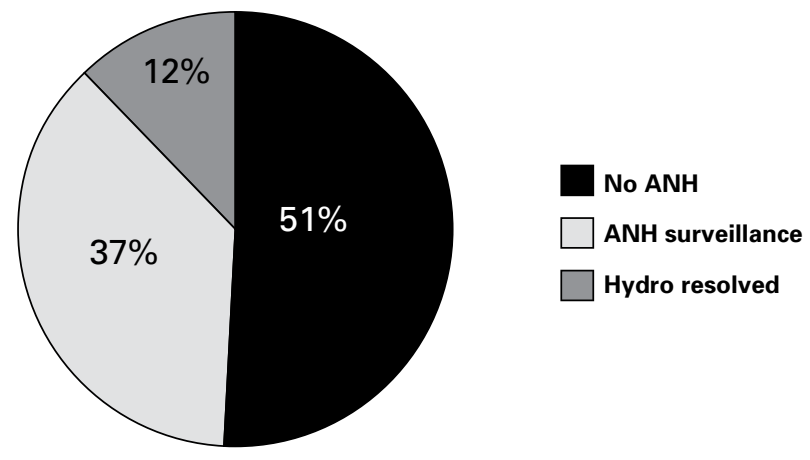

Fig. 1. Pie graph depicting proportion of all patients who underwent pyeloplasty, with a breakdown of the prenatal ANH history in symptomatic patients. ANH: antenatal hydronephrosis.

4). Their mean initial differential function was $38 \%$ on the affected side (range: $25 \%-48 \%$ ).

Eight patients in our cohort had an initial function of $25 \%$ or less prior to surgery. Of these 8 patients, 7 presented with symptoms, with no history of $\mathrm{ANH}$, and only 1 was asymptomatic.

\section{Discussion}

Pediatric pyeloplasties remain a common surgical intervention for the pediatric urologist. Despite the prevalence of screening prenatal ultrasounds, most pyeloplasties at our institution were preformed due to symptoms. Although the effectiveness of postnatal follow-up is beyond the scope of this paper, we feel that our data are important.

Most of the current published data is extremely liable to surgeon's bias due to the use of surgical intervention as a primary end-point of UPJ follow-up. ${ }^{1}$ Many manuscripts have been written extolling the success of conservative management, whereby observation is undertaken until images demonstrate deterioration (progression of drainage curves, loss of differential renal function, or increase in hydronephrosis). ${ }^{1,4}$ Unfortunately, the other main conclusion of all of these studies is that we are unable to accurately predict which patients will suffer a deterioration in function or develop a complication (urinary tract infection, Dietl's crisis, stone). ${ }^{1,5}$ Both ultrasound and nuclear radiography have many variables which affect their interpretations, and thus, reduce their accuracy. ${ }^{6-8}$

Therefore, we focused on those patients who had undergone surgery and compared their indications. This is fundamentally different from most data, as it did not attempt to predict outcomes. Instead, we wanted a better idea of where our surgery was coming from. We felt that despite all of the resources attempting to predict postnatal radiographic imag- ing, a significant number of our patients simply presented with the classic indications for surgical intervention.

Our results confirm our hypothesis - most patients who underwent a pyeloplasty at our institution were symptomatic, and therefore, did not benefit from early intervention secondary to antenatal screening. This was either due to antenatal hydronephrosis not being detected, the resolution of hydronephrosis on follow-up imaging, or the development of symptomatic indications for surgery while undergoing surveillance. This may also be due to a bias in our postnatal monitoring, but this effect may be minor at best.

In keeping with previous literature, the 4 most common symptoms include: pain (42\%), infections (32\%), renal insufficiency $(7 \%)$, and stones $(7 \%)$. This distribution did not vary between our 2 patient populations.

Surgeons have conservative thresholds for intervention for patients undergoing surveillance after $\mathrm{ANH}$. The authors adhere to commonly accepted criteria for asymptomatic intervention, which includes an initial differential renal func-

Table 3. Prevalence of symptoms present at surgery in patients who had a symptomatic indication for surgery

\begin{tabular}{lc}
\hline Symptoms & $\mathbf{n}(\%)$ \\
\hline No. symptomatic patients & $43(60 \%)$ \\
Pain & $18(42 \%)$ \\
Dietl's crisis & $3 / 18$ \\
Trauma & $1 / 18$ \\
UTI & $14(32 \%)$ \\
Abscess & $1 / 14$ \\
Sepsis & $1 / 14$ \\
Renal insufficiency & $3(7)$ \\
Stones & $3(7)$ \\
Failure to thrive & $3(7)$ \\
Urinary incontinence & $2(5)$ \\
\hline
\end{tabular}

UTI: urinary tract infection. 
tion $<40 \%$ with impaired drainage (as indicated by T1/2 $>20$ minutes), worsening renal function, deterioration of drainage curves, or increasing hydronephrosis on ultrasound. ${ }^{1}$

Eight patients underwent surgery secondary to a decrease in function on nuclear renography, and only $50 \%$ returned to $90 \%$ of their baseline function. This is consistent with many published reports. ${ }^{9,10}$ Unfortunately, we were not able to identify any characteristics to help differentiate these patients from those who maintained their baseline function.

It is very reassuring that there was no statistically significant difference in postoperative outcomes between those patients with symptomatic indications and those who were asymptomatic. Furthermore, our MAG-3 data suggest that most symptomatic patients retained good ipsilateral function, implying that renal function did not deteriorate. However, we were significantly concerned about our patients who underwent observation with an asymptomatic documented loss of function on MAG-3 scans.

There are several significant limitations to our study. We did not have a denominator for the total number of patients who underwent surveillance after detection of $\mathrm{ANH}$; therefore, we were unable to calculate the overall rate of intervention. This was especially obvious when looking at our patients with grade 3-4 hydronephrosis, and we were unable to calculate the incidence of symptoms and intervention and compare to those who were successfully managed conservatively. However, we did not design this study to specifically discuss our screening outcomes. Secondly, the grading systems used for both ultrasound and MAG-3 were very subjective and did not lend themselves to statistical calculation. Therefore, we were unable to accurately compare imaging characteristics of our groups. Finally, as with many papers on this topic, surveillance patterns and surgical indications in an asymptomatic patient are very subjective and prone to bias.

\section{Conclusion}

Despite a great deal of time, effort, and resources, our current preventive strategies are unable to prevent morbidity in most of our patients who had undergone pyeloplasty. Most patients who underwent surgery for UPJO presented with de-novo symptoms, and had no prior evidence of $\mathrm{ANH}$. Several patients developed symptoms while under surveillance, and several had complications after their radiography was normal and they were discharged from regular screening. Although our lack of a denominator negates our ability to discuss surveillance strategies, this was not our intention. We simply wanted to reinforce the notion that symptoms secondary to a UPJO remain a significant indication for pyeloplasty, despite ubiquitous screening.

Competing interests: Dr. Metcalfe, Dr. Assmus and Dr. Kiddoo declare no competing financial or personal interests.

This paper has been peer-reviewed.

\section{References}

1. Nguyen HT, Herndon CA, Cooper C, et al. The Society for Fetal Urology consensus statement on the evaluation and management of antenatal hydronephrosis. J Pediatr Urol 2010;6:212-31.

2. Karnak I, Woo LL, Shah SN, et al. Results of a practical protocol for management of prenatally detected hydronephrosis due to ureteropelvic junction obstruction. Pediatr Surg Int 2008;25:61-7. http://dx.doi. org/10.1007/s00383-008-2294-6

3. Alladi A, Agarwala S, Gupta AK, et al. Postnatal outcome and natural history of antenatally-detected hydronephrosis. Pediatr Surg Int 2000;16:569-72. http://dx.doi.org/10.1007/s003830000416

4. Lee RS, Cendron M, Kinnamon DD, et al. Antenatal hydronephrosis as a predictor of postnatal outcome: $A$ meta-analysis. Pediatrics 2006; 118:586-93. http://dx.doi.org/10.1542/peds.2006-0120

5. Koff SA, Campbell KD. The nonoperative management of unilateral neonatal hydronephrosis: Natural history of poorly functioning kidneys. J Urol 1994;152:593-5.

6. Caglar M, Gedik GK, Karabulut E. Differential renal function estimation by dynamic renal scintigraphy: Influence of background definition and radiopharmaceutical. Nud Med Commun 2008;29:1002-5. http:// dx.doi.org/10.1097/MNM.0b013e32830978af

7. Gordon II, Piepsz AA, Sixt RR. Guidelines for standard and diuretic renogram in children. Eur J Nucl Med Mol Imaging 2011;38:1175-88. http://dx.doi.org/10.1007/s00259-011-1811-3

8. Snodgrass WT. Pediatric Urology. Springer; 2013. http://dx.doi.org/10.1007/978-1-4614-6910-0

9. Chertin B, Rolle U, Farkas $A$, et al. Does delaying pyeloplasty affect renal function in children with a prenatal diagnosis of pelvi-ureteric junction obstruction? BJU Int 2002;90:72-5. http://dx.doi. org/10.1046/i.1464-410X.2002.02829.x

10. Palmer $L S$, Maizels $M$, Cartwright $P C$, et al. Surgery versus observation for managing obstructive grade 3 to 4 unilateral hydronephrosis: A report from the Society for Fetal Urology. I Urol 1998;159:222-8. http://dx.doi.org/10.1016/S0022-5347(01)64072-2

Correspondence: Dr. Peter D. Metcalfe, Associate Professor, University of Alberta, Stollery Children's Hospital, 2 C3.79 WMC 8440-112 St., Edmonton, AB T6G 2B7; pmetcalf@ualberta.ca 